\title{
Detection of Brain Tumor in Early Stage is Crucial
}

\author{
Sudipta Roy ${ }^{1}$ and Samir Kumar Bandyopadhyay ${ }^{2}$ \\ ${ }^{1}$ Department of Computer Science \& Engineering, University College of Technology, University of Calcutta, India \\ ${ }^{2}$ Advisor to Chancellor, JIS University, India
}

Submission: January 21, 2018; Published: March 16, 2018

*Corresponding author: Sudipta Roy , Department of Computer Science \& Engineering, University College of Technology, University of Calcutta, India, Email: Samir.bandyopadhyay@jisgroup.org

Abstract

A brain tumor is the most common and widespread disease among these brain diseases. Early and accurate diagnosis of brain lesion is vital for determining specific treatment and prognosis. However, the diagnosis is a very challenging task and can only be performed by specialists in neuroradiology.

Keywords: Brain tumor; Lesion; Neuroradiology

\section{Introduction}

Brain tumor, stroke, hemorrhage and multiple sclerosis (MS) disease are the life threatening diseases in both male and female. A brain tumor is the most common and widespread disease among these brain diseases. The worldwide cancer incidence of brain tumor is 3.4 per 100,000 people (men: 3.9 per 100,000, women: 3.0 per 100,000 . A total of 256,213 affected worldwide $(139,608$ men and 116,605 women). The trend of new cases is rising and 189,582 sufferers worldwide. Every day about 700 people is diagnosed with a brain tumor [1]. 15 million people are affected by stroke and hemorrhage; of this 5 million die and another 5 million (2002 estimates) are permanently disabled. Today over 2,500,000 people around the world have MS [2].

Early and accurate diagnosis of brain lesion is vital for determining specific treatment and prognosis. However, the diagnosis is a very challenging task and can only be performed by specialists in neuroradiology. There are at least two specialists required to examine and confirm of each medical report on imaging investigations. Any difficulty may necessitate invasive tests such as biopsy and surgery. Currently, the standard lesion pathological classification is based on histological examination of tissue samples through biopsy.

Therefore, radiologists are continuously seeking for greater diagnosis accuracy by the modern medical imaging system. According to quantitative analysis of computer-aided diagnosis (CAD), it may aid radiologists in the interpretation of the medical images. Recent studies showed that CAD could help to improve the diagnostic accuracy of radiologists, lighten their increasing workload, reduce misinterpretation due to fatigue or overlooked and improved inter- and intra-reader variability [3]
MRI of brain image analysis is the task of identifying image components (pixels) into relevant anatomical components or describing the structural and intensity changes regarding the underlying functional process. The knowledge of the location, size, and shape of different anatomical structures is a fundamental step in understanding and analyzing medical images. Automated detection and analysis of normal and abnormal tissues in MR images with high accuracy and low error allow us to do more natable measurement than qualitative visual assessment.

a) Detection and localization of brain tissues to healthy anatomical structures are useful in planning radiological treatments and surgeries.

To develop CAD system that separates the normal and abnormal lesions from brain region.

a) Analysis of the size and volume of the segmented brain structures can be used to find characteristics or markers of neurological brain disorders.

b) Growth and inhomogeneity is determined by analyzing of segmented structures.

c) CAD analysis of abnormalities and categorization demonstrated to improve efficiency and accuracy of clinical practice hence it decreases the risk of misdiagnosis and mismanagement.

Automatic segmentation of medical images can facilitate an imaging-based diagnosis, providing aid to surgery and treatment planning. Computer technology has had a tremendous impact on medical imaging. Computer-Aided Diagnosis (CAD) is a relatively young interdisciplinary technology combining 
elements of digital image processing with medical image processing. CAD techniques in X-ray, Mammogram, and MRI and Ultrasound diagnostics yield a great deal of information, which the radiologist has to analyze and evaluate comprehensively in a short time. The interpretation of medical images, however, is still almost exclusively the work of humans but in the next decades, this change is expected. The computer is used to more image interpretation, and the research area is called CAD.

\section{Benefits of using MRI}

MRI is the most frequently used neuroimaging technique for the evaluation and follow-up a review of patients with brain abnormalities for many reasons [4]. It does not use ionizing radiation like computed tomography (CT), single photon emission computed tomography (SPECT), and positron emission tomography (PET) studies. MRI has following list of benefits:

MRI contrast resolution is higher than the other techniques, making it preferable for detecting small lesions and isodense lesions on unenhanced CT.

a. It is more sensitive than CT to detect lesion enhancement. The ability of MRI devices to generate images in the sagittal, axial and coronal planes provides better localization of a lesion in the 3D space of the brain and allows structures involved by the abnormalities to be more clearly delineated.

b. MR imaging eliminates the beam-hardening artifact produced by the skull base on CT, making it better for evaluating lesions in the posterior fossa and the inferior frontal and temporal lobes.

c. The development of MR spectroscopy, MR diffusion imaging, and MR perfusion imaging now permits evaluation of tumor physiology with MR scanners.

d. The acquisition of both functional and anatomical information about the tumor during the same scan may be the most important benefit of MR imaging [4] in 2001.

e. It can noninvasively record brain signals (of humans and other animals) without risks of radiation inherent in other scanning methods, such as CT or PET scans.

In short, it is stated that the T1 MR images have brighter pixels for white matter (WM), darker for gray matter (GM), and almost black for cerebrospinal fluid (CSF). The T1 images show abnormality with larger intensity value than normal tissue. Therefore, some lesions in the WM areas can look alike GM in T1 images due to the increase of water. Besides, the pixels with muscle tissue appear brighter than for fat. Almost the opposite intensity contrast will be expected in T2 images. The WM is less fluid-based. Thus the pixels with mostly this tissue class will appear white in $\mathrm{T} 1$ and dark in $\mathrm{T} 2$, which corresponds to high and low-intensity values, respectively. In the case of GM, it appears darker in T1 and brighter in the T2 images. Finally, the CSF shows a small peak but also a big lobe that almost overlaps all the classes. In proton density (PD) images white matter is brighter than gray matter, and gray matter is brighter than CSF. $\mathrm{PD}, \mathrm{T} 2$, and T1 type of MRI with sarcoma brain abnormality is shown in Figure 1.

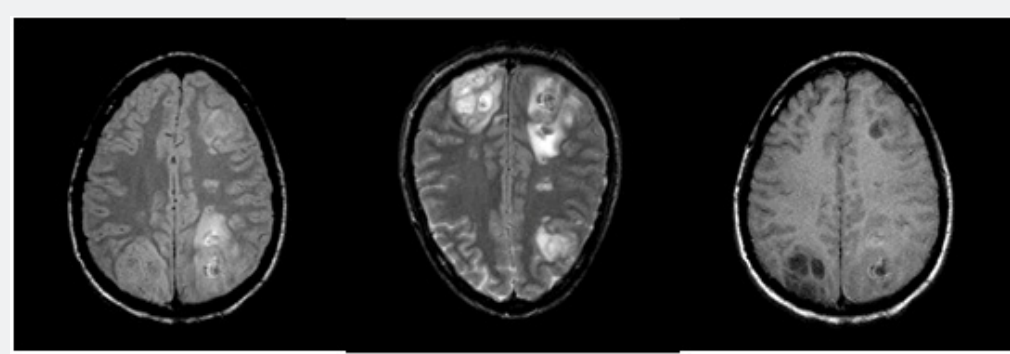

PD
$\mathrm{T} 2$
$\mathrm{T} 1$

Figure 1: PD, T2, and T1 type of MRI with sarcoma type of brain tumor.

\section{Proposed Method}

The proposed techniques implemented based on brain MRI images consisting of normal and abnormal from a real human brain MRI dataset. The dataset used consists of axial, T2, T1, and PD MR Brain Images. These images were collected from the Harvard Medical School [5] website used for normal and abnormal brain images: (a) tumors, (b) strokes, (c) hemorrhage, and (d) MS. Standard simulations from brain web database [6] include parameter setting fixed to 3 modalities (T1, T2, and PD), five slice thicknesses $(1,3,5,7$ or $9 \mathrm{~mm})$, and defining a volume $(\mathrm{x}, \mathrm{y}, \mathrm{z})$. Tissue classes include GM, WM, and CSF but also muscle, fat or skin. The brain model used to generate the simulations can also be employed as ground truth. Another public domain dataset is used to conducting similar research. Easy Analytic Software, Inc (EASI) MRI database for different brain abnormality MR images [7]. All mentioned dataset are used to evaluate the performance.

An abnormal lesion is a lesion of the brain tissues that suppress and occupy the normal tissue lesions area. Various factors that lead to abnormal brain lesion development include brain injuries, multiple sclerosis, hemorrhage, stroke, vascular disorders and brain tumors. Diagnosis of brain lesions is highly variable depending on the type of lesion, the age and health condition of the patient, and how effective treatments are for the patient. 
A brain tumor is a cluster of abnormal cells due to loss of normal aging and cell death. It may occur in any person at almost any age. It may even change from one treatment session to the next, but its effects may not be the same for each person. Brain tumors appear at any location, in different image intensities, can have a variety of shapes and sizes. Brain tumors can be malignant or benign. In this research work five major tumors glioma, meningioma, metastatic adenocarcinoma, metastatic bronchogenic carcinoma, and sarcoma types of tumors are used. Gliomas are a group of tumors that arise in the central nervous system. MR imaging is currently the method of choice for early detection of a brain tumor in the human brain. Low-grade gliomas and meningiomas are benign tumors. Glioblastoma multiforme is a malignant tumor and may arise anywhere in the brain. Metastatic adenocarcinoma and Metastatic bronchogenic carcinoma tumors are the most common type of brain tumors (30\% of all) and are usually malignant one. Sarcoma arises in the nearer to surrounding structures of the brain. According to World Health Organization (WHO), there are 126 types of different brain tumors of which many of them arise from structures intimately associated with the brain such as tumors of the covering membranes to the posterior fossa. Figure 2 shows the five major types of tumors in MRI images with arrows.

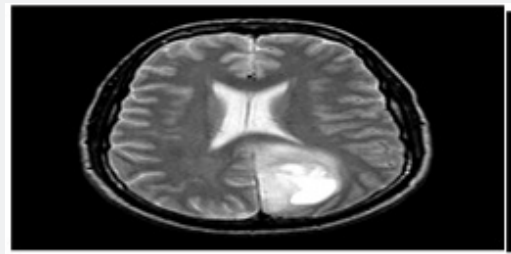

(a)

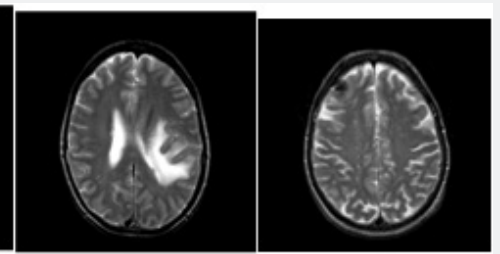

(b)

(c)

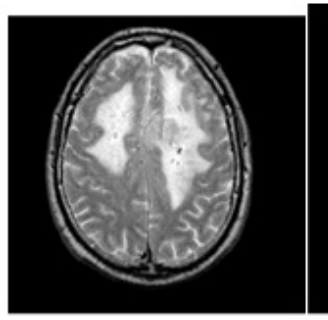

(d)

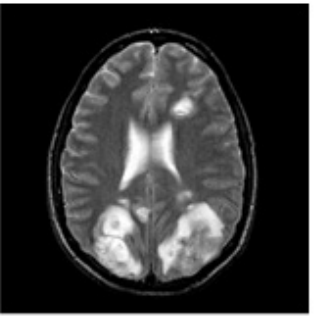

(e)

Figure 2: Five major brain tumors in MRI: a) Glioma, b) Meningioma, c) Metastatic adenocarcinoma, d) Metastatic bronchogenic carcinoma, and e) Sarcoma.

\section{Conclusion}

Brain tumors form the second most cause of cancer-related deaths in children and adults. An adaptive neuro-fuzzy inference system is used to classify the five major types of brain tumors: 1) Glioma, 2) Meningioma, 3) Metastatic adenocarcinoma, 4) Metastatic bronchogenic carcinoma, and 5) Sarcoma for computerized classification. This classification can help the physicians to identify the type of brain tumors for further treatment and diagnosis.

\section{References}

1. http://globocan.iarc.fr/Default.aspx
2. http://www.msif.org

3. Ei SA, EI D, Tamer H, Abdel B (2010) Hybrid intelligent techniques for MRI brain images classification. Digital Signal Processing 20(2): 433441.

4. Ricci PE, Dungan DH (2001) Imaging of low- and intermediate-grade gliomas. Semin Radiat Oncol 1(2):103-112.

5. http://www.med.harvad.edu/AANLIB/home.html

6. http://brainweb.bic.mni.mcgill.ca/brainweb/

7. http://www.easidemographics.com/cgi-bin/dbmri.asp

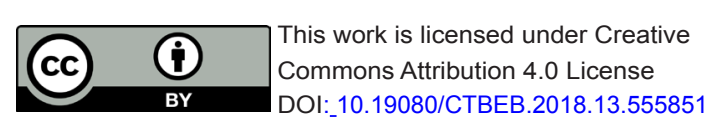

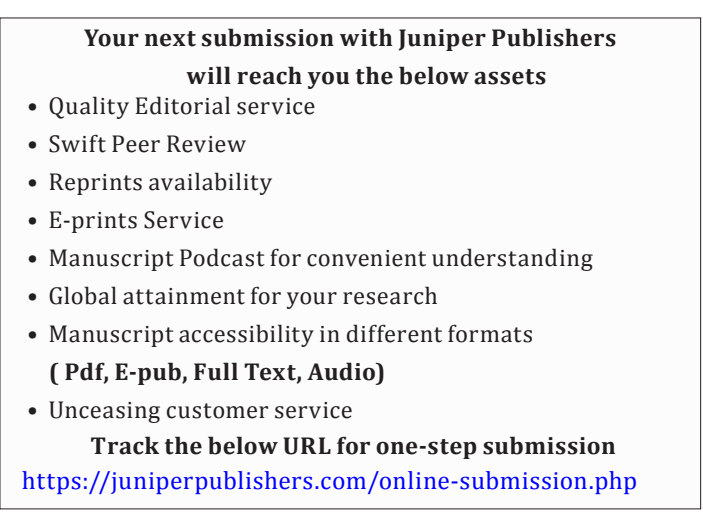

